\title{
Clinical Vignettes
}

\section{Liver Failure after Hydroxycut'T Use in a Patient with Undiagnosed Hereditary Coproporphyria}

\author{
Stephanie Haimowitz, MD ${ }^{7}$, Jennifer Hsieh, MD², Marina Shcherba, MD², and \\ Yelena Averbukh, $\mathrm{MD}^{1,2}$ \\ 'Albert Einstein College of Medicine, Bronx, NY, USA; ${ }^{2}$ Montefiore Medical Center, Bronx, NY, USA.
}

We report the case of a young male presenting with cholestatic liver failure. After an extensive workup, the etiology of the liver failure was determined to be due to hereditary coprophorphyria (HCP). The inciting event was the use of Hydroxycut ${ }^{\mathrm{TM}}$, an over-the-counter supplement to promote weight loss that has been reported to cause oxidative liver injury in vulnerable populations. Although $\mathrm{HCP}$ is a rare cause of cholestatic liver failure, it is treatable if diagnosed correctly and in a timely manner. In this clinical vignette, we discuss a case that highlights the genetic susceptibility to disease that can be unmasked by environmental exposures. We also review the relevant literature on Hydroxycut ${ }^{\mathrm{TM}}$ and how it can affect hepatic function.

KEY WORDS: porphyria; liver failure; herbal supplement use.

J Gen Intern Med 30(6):856-9

DOI: $10.1007 / \mathrm{s} 11606-014-3153-x$

(c) Society of General Internal Medicine 2015

\section{INTRODUCTION}

Hereditary coproporphyria (HCP), an acute hepatic porphyria, is the result of a mutation in the $C P O X$ gene, which encodes coproporphyrinogen oxidase (CPOX), the sixth enzyme in the heme biosynthetic pathway. The enzyme deficiency causes subsequent accumulation of the intermediate coproporphyrin III. The disease is inherited in an autosomal dominant fashion, but is commonly latent before puberty. ${ }^{1}$ Patients present for medical evaluation once they manifest symptoms such as abdominal pain, nausea, vomiting, rash, or seizures. Acute attacks can be precipitated by drugs (a majority of which remain unidentified), environmental stressors, or dietary changes. Because the disease is rare (combined incidence for all forms of the disease 1:20,000) and symptoms are often non-specific, the diagnosis of porphyria is difficult to make. ${ }^{2}$ Thus, for any patient presenting with liver dysfunction and workup incongruous with more common causes, a certain degree of suspicion by the medical practitioner is required to establish the correct diagnosis of porphyria.

Received July 3, 2013

Revised November 19, 2013

Accepted December 12, 2014

Published online February 10, 2015

\section{CASE}

A 23-year-old male with no significant past medical history presented with a photosensitive rash, nausea, vomiting, and abdominal pain for 4 days. He denied changes in diet and illicit drug or alcohol use, but admitted to using the over-the-counter (OTC) weight-loss supplement Hydroxycut ${ }^{\mathrm{TM}}$ for the past few months. The remaining review of systems was negative. He appeared toxic, with a temperature of $102^{\circ} \mathrm{F}$, heart rate of 125 beats per minute, marked jaundice, right upper quadrant abdominal tenderness, and a hyperpigmented macular rash on the extensor surface of his upper extremities and dorsum of his hands. His body mass index (BMI) was 25 . Notable laboratory tests included a white blood cell count (WBC) of 40.3/1, total bilirubin of $24.4 \mathrm{mg} / \mathrm{dl}$ (normal $0.2-1.3 \mathrm{mg} / \mathrm{dl}$ ), conjugated bilirubin of $17.5 \mathrm{mg} / \mathrm{dl}$ (normal $<0.3 \mathrm{mg} / \mathrm{dl}$ ), alkaline phosphatase of $91 \mathrm{U} / 1$ (normal 38-126 U/1), aspartate aminotransferase (AST) 194 U/1 (normal 5-35 U/1), alanine aminotransferase (ALT) $92 \mathrm{U} / 1$ (normal 7-56 U/l), and international normalized ratio (INR) of 1.5 (normal $0.8-1.2$ ). Imaging was done to investigate for common causes of cholestatic disease. Abdominal ultrasound, computerized tomography (CT scan) of the abdomen and pelvis, and magnetic resonance cholangiopancreatography (MRCP) were unremarkable. A full infectious disease workup, which included bacterial, mycobacterial, viral, and parasitic etiologies, was also nondiagnostic. Persistent leukocytosis with a monocytic predominance and continuous fevers prompted additional workup for a hematologic malignancy, which also did not yield a cause. Due to acute cholestasis of unclear etiology, the patient underwent a liver biopsy that showed cholestasis with a neutrophilic infiltrate in the lobules and focal centrilobular sinusoidal dilatation with dropout of hepatocytes, suggesting druginduced liver injury.

Over the next 2 months, the patient continued to deteriorate, with a hospital course complicated by acute kidney injury and spontaneous bacterial peritonitis requiring multiple abdominal washouts, despite supportive care and prolonged discontinuation of Hydroxycut ${ }^{\mathrm{TM}}$. His history and hospital course were reexamined. The upper extremity photosensitive rash along with his young age and persistent cholestatic liver failure suggested that he may have a rare and genetic cause for disease. The possibility of porphyria was entertained and appropriate investigative studies were performed. 
Measurement of plasma porphyrin showed a total porphyrin level of $100.2 \mathrm{mcg} / 1$ (normal 1.0-5.6 mcg/l) and a coproporphyrin level of $93.6 \mathrm{mcg} / 1$ (normal $<0.9 \mathrm{mcg} / \mathrm{l}$, levels less than 3 times normal are associated with medication use; levels greater than 5 times normal are associated with porphyria attacks). Further genetic testing identified a frameshift mutation in the $C P O X$ gene (c.119delA). He was found to be heterozygous for this mutation. Once the diagnosis of hereditary coproporphyria had been established, he was treated with hemin with good clinical response and resolution of symptoms.

\section{DISCUSSION}

Porphyrias are a heterogeneous group of metabolic syndromes that result from abnormal enzymatic activity in the heme biosynthetic pathway. There are eight enzymes involved in the synthesis of heme, and a deficiency in one of these enzymes leads to the accumulation of the porphyrin intermediates (Fig. 1). ${ }^{3}$ These intermediates are highly reactive oxidants and can be neurotoxic, leading to neurovisceral symptoms such as nausea, vomiting, abdominal pain, and seizures. ${ }^{2,3}$ Porphyrias can be classified as either hepatic or erythropoietic. The neurovisceral symptoms in hepatic porphyrias, such as acute intermittent porphyria and hereditary coproporphyria, result from abnormal heme synthesis in the liver, which is needed for the production of the hepatic CYP enzymes. This typically manifests during childhood, but individuals may remain asymptomatic in the absence of triggering factors, as in the case of our patient. The most common presenting symptoms in patients with HCP include abdominal pain, nausea, and vomiting. In addition to abdominal pain, a symptom that is found in $80 \%$ of cases, our patient also presented with jaundice, a finding reported in only $20 \%$ of cases. ${ }^{4}$ Rash and photosensitivity can also be seen in HCP, but are not as common as in erythropoietic or cutaneous porphyrias. Though leukocytosis is not a common presenting finding in patients with acute attacks of HCP, it has been documented in the literature with white cell count elevation ranging from 14,000 to 30,000 cells $/ \mathrm{mm}^{3}$.

There are a variety of biochemical tests available to test for porphyrias; however, none of these tests are sensitive or specific for acute porphyrias. The validity of the tests depends on the porphyria type, test used, stage of illness, and timely evaluation of the sample. For example, when assessing the presence of urinary porphyrins, the collected urine sample must not be exposed to light, as levels can decrease by $50 \%$ in $24 \mathrm{~h}$, leading to false-negative results. ${ }^{8}$ Conversely, urinary porphyrin levels may be elevated in conditions unrelated to an acute porphyria attack such as sepsis and renal or hepatic dysfunction. Any extent of liver dysfunction can reduce biliary excretion of coproporphyrin, causing urinary coproporphyrin excretion to increase. ${ }^{8}$ As a result, an elevation in the urinary porphyrin level can be erroneously interpreted as being secondary to an abnormality in heme synthesis. Thus, caution should be used when interpreting the biochemical data and should always be considered in the context of the specific patient. The specific heme intermediate or intermediates that are elevated aid in the diagnosis of the particular porphyria. Measurement of plasma porphyrins is typically used in patients with cutaneous symptoms, whereas measurement of fecal porphyrin levels is more commonly used in patients with only neurovisceral symptoms. ${ }^{9}$ A definitive diagnosis can be made via genetic testing and mutation analysis for specific abnormalities. Once the particular defect has been identified, these DNA studies can be used to identify at-risk family members so that appropriate counseling can be provided. ${ }^{8}$ Since our patient presented with a rash, the plasma porphyrin levels were measured. The elevation of coproporphyrin in the plasma made the diagnosis of HCP the most likely candidate. The DNA testing that followed was targeted toward the known mutations in the CPOX gene that have been implicated in reported cases of HCP.

Treatment of an acute attack should always include supportive and symptomatic interventions. These include intravenous hydration, pain management with a narcotic, correction of electrolyte abnormalities, glucose administration, and, most

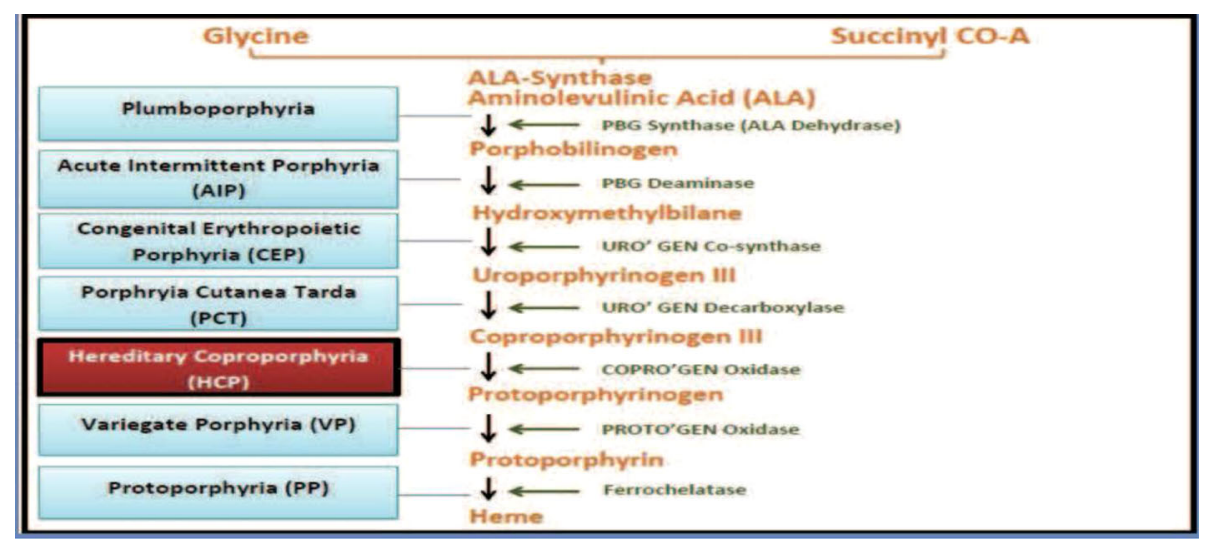

Fig. 1 Diagram of the heme biosynthetic pathway. Horizontal arrows denote the enzyme used in each step. Horizontal lines refer to the porphyria that results from the corresponding enzyme deficiency. 
importantly, a review of the patient's current medications and discontinuation of any that may exacerbate the condition. In order to correct the underlying pathophysiologic disturbance present during an acute porphyria attack, intravenous hemin is administered to replenish some of the depleted heme supply and also to serve as negative feedback to suppress aminolevulinic acid synthase (ALAS) activity, the ratelimiting step in heme biosynthesis. ${ }^{8}$ The typical dose is $3-$ $4 \mathrm{mg} / \mathrm{kg}$ of hemin daily for 4 days. Intravenous hemin is generally well tolerated and is considered safe to use during pregnancy, and side effects are uncommon. However, side effects of fever, malaise, hemolysis, anaphylaxis, and circulatory collapse have been reported. Therefore, patients should be carefully observed throughout treatment so adverse reactions can be identified early and the appropriate intervention implemented if necessary.

There are many known triggers for an acute porphyria attack, including infection, hormonal fluctuations, fasting, smoking, or exposure to porphyrinogenic drugs. Some of the more commonly prescribed medications with porphyrinogenic potential include erythromycin, nitrofurantoin, and dimenhydrinate (generic form of Dramamine) ${ }^{10}$ Recurrent attacks are more common in females than in males. Hormonal fluctuations, as is seen in the menstrual cycle or during pregnancy, are well-documented inducers of acute porphyria attacks. Although the exact mechanism is unknown, it seems that progesterone increases oxidative stress and formation of reactive oxygen species by inducing activity of many Krebs Cycle enzymes, resulting in tissue damage and disruption of mitochondrial integrity. ${ }^{11}$ Attacks can be triggered by either the induction of the enzyme delta-aminolevulinate synthase 1 (ALAS1) directly or increasing the need for hepatic heme synthesis. The induction of hepatic CYP enzymes by various inciting agents is the pathophysiologic mechanism underlying many attacks, as this leads to an increase in hepatic heme turnover. ${ }^{2}$ Hydroxycut ${ }^{\mathrm{TM}}$ is an OTC medication used for weight loss that has been associated with hepatotoxicity in 23 case reports, perhaps due to increased oxidative stress. Upon cessation of the drug, liver function improved within 7 weeks for most patients and often normalized by week $14 .{ }^{12-}$

${ }^{14}$ In the case of our patient, Hydroxycut ${ }^{\mathrm{TM}}$ use was the cause of initial liver injury leading to fulminant failure in the setting of underlying porphyria.

With the public's increasing awareness of the growing obesity epidemic and its many negative impacts on public and personal health, herbal remedies and weight loss supplements continue to grow in popularity. Labeling a drug as an OTC supplement or herbal remedy often gives the impression to the general public that the product is safe. However, due to limited large-scale research trials focused on these herbal ingredients, their effects and potential deleterious interactions with other drugs and medical conditions are not always well established. As a result, it is often not until these harmful effects are seen that a retrospective study elucidates these hazards. ${ }^{12,13}$ For example, there are many components in
Hydroxycut ${ }^{\mathrm{TM}}$ that are potentially hepatotoxic. Components of various Hydroxycut ${ }^{\mathrm{TM}}$ formulations for which hepatotoxicity have been described include Camellia sinensis and hydroxycitric acid. ${ }^{13,15}$ The most likely pattern of injury is hepatocellular; $5,13,16,17$ however, there are reports that describe a cholestatic pattern of liver injury. ${ }^{14,18,19}$ In 2009, the US Food and Drug Administration (FDA) released a statement warning consumers to stop using Hydroxycut ${ }^{\mathrm{TM}}$ as there were 23 case reports describing various sequelae ranging from transient elevated liver tests to fulminant liver failure requiring transplantation. ${ }^{20}$ While a single ingredient may not demonstrate significant hepatotoxic effects in animal models, the interaction among various components along with preexisting medical conditions can have profound toxic effects. For example, in our case, Hydroxycut ${ }^{\mathrm{TM}}$ was the inciting agent for the patient's acute porphyria attack. The hepatotoxicity, while reversible in a person with normal heme metabolism after discontinuation of the offending agent, in an individual with an underlying metabolic disorder, the direct hepatotoxic effects also had a porphyrinogenic component due to the resulting increased oxidative stress and increased hepatic heme turnover. While the formulation of Hydroxycut ${ }^{\mathrm{TM}}$ has since changed and there does not appear to be a large number of reports of liver injury associated with the newer formulations, some of the ingredients present in the earlier formulation are being used in newer weightloss supplements. Thus, these newer weight loss supplements may also have the potential to unmask disease in susceptible patients.

\section{CONCLUSION}

In this case, although Hydroxycut ${ }^{\mathrm{TM}}$ was thought to be the culprit initially, the gravity of the patient's illness and lingering decompensation despite prolonged discontinuation of Hydroxycut $^{\mathrm{TM}}$ led us to believe that there may have been another underlying etiology that was contributing to the patient's clinical condition. The importance of reevaluation of the initial presentation of this patient was very important for the final diagnosis. The connection between the presence of a rash at initial presentation and abdominal symptoms in a young male was not recognized as a sign of underlying hereditary disorder, but was attributed to the drug effect. However, upon further investigation and reevaluation of the data, including the photosensitive nature of the rash, abdominal complaints, and liver disease, the physicians explored other important but rare diagnoses. Further testing was done that ultimately led to the diagnosis of HCP, which accounts for a very small percentage of all patients presenting with cholestatic liver failure. Therefore, it is imperative that physicians be cognizant of notable side effects of frequently used herbal supplements, while also remaining suspicious of rarer diagnoses with an unexpected clinical course. 
Acknowledgements: This clinical vignette was previously presented as a poster presentation at the SGIM national conference in Orlando in May 2012.

Conflict OfInterest: The authors declare that they have no conflicts of interest.

Corresponding Author: Stephanie Haimowitz, MD; Albert Einstein College of Medicine, 85 Cherry Lane, Teaneck, NJ 07666, USA (e-mail: stephhaimowitz@gmail.com).

\section{REFERENCES}

1. Wintrobe MM, Greer JP. Wintrobe's clinical hematology. 12th ed. Philadelphia: Wolters Kluwer Health/Lippincott Williams \& Wilkins; 2009.

2. Puy H, Gouya L, Deybach JC. Porphyrias. Lancet. 2010;375(9718):924-937.

3. Gonzalez-Arriaza HL, Bostwick JM. Acute porphyrias: a case report and review. The American Journal of Psychiatry. 2003;160(3):450-459.

4. Kapetanos D, Xiarchos P, Kapetis E, Avgerinos A, Mias A, Kokozidis G, Kitis G. Hereditary corpoporphyria presenting with deep jaundice and photosensitivity. Annals of Gastroenterology. 2007;14(4)

5. Dahlgren M, Khosroshahi A, Stone JH. A 22-year-old woman with severe headaches, vomiting, and tonic-clonic seizures. Arthritis Care \& Research. 2011;63(1):165-171.

6. Karcz A, Farkas PS. Acute porphyria in the emergency department. The Journal of Emergency Medicine. 1989;7(3):279-285.

7. Hartstein G. The Patient with Porphyria. In: Frost EM, ed. Preanesthetic Assessment 1: Birkhäuser Boston; 1987:166-176

8. Jain G, Bennett JI, Resch DS, Godwin JE. Schizoaffective disorder with missed diagnosis of acute porphyria: a case report and overview. The primary care companion to CNS disorders. 2011;13(6)
9. Foran SE, Abel G. Guide to porphyrias. A historical and clinical perspective. American Journal of Clinical Pathology. 2003;119:S8693.

10. PF. http://db.porphyriafoundation.com/forms/drug-list.lasso

11. Schmidt M, Schmitz HJ, Baumgart A, Guedon D, Netsch MI, Kreuter MH, et al. Toxicity of green tea extracts and their constituents in rat hepatocytes in primary culture. Food ChemToxicol. 2005;43:307-14.

12. Dara L, Hewett J, Lim JK. Hydroxycut ${ }^{\mathrm{TM}}$ hepatotoxicity: a case series and review of liver toxicity from herbal weight loss supplements. World Journal of Gastroenterology: WJG. 2008;14(45):6999-7004.

13. Sharma T, Wong $\mathbf{L}$, Tsai $\mathbf{N}$, Wong RD. Hydroxycut ${ }^{\mathrm{TM}}((\mathrm{R}))$ (herbal weight loss supplement) induced hepatotoxicity: a case report and review of literature. Hawaii Medical Journal. 2010;69(8):188-190.

14. Stevens T, Gadri A, Zein NN. Two patients with acute liver injury associated with use of the herbal weight-loss supplement Hydroxycut ${ }^{\mathrm{TM}}$. Annals of Internal Medicine. 2005; 142(6):477-478.

15. Actis GC, Bugianesi E, Ottobrelli A, Rizzetto M. Fatal liver failure following food supplements during chronic treatment with montelukast. Digestive and Liver Disease: official journal of the Italian Society of Gastroenterology and the Italian Association for the Study of the Liver. 2007;39(10):953-955.

16. Bonkovsky HL. Hepatotoxicity associated with supplements containing Chinese green tea (Camellia sinensis). Ann Intern Med. 2006;144(1):68-71.

17. Pedrós C, Cereza G, Garcia N, et al. Liver toxicity of Camellia sinensis dried etanolic extract. Med Clin (Barc). 2003;121(15):598-9.

18. Chuah LO, Yeap SK, Ho WY, Beh BK, Alitheen NB. In vitro and in vivo toxicity of garcinia or hydroxycitric acid: a review. Evidencebased Complementary and Alternative Medicine: eCAM. 2012;2012:197920.

19. Pushpalatha T, Reddy PR, Reddy PS. Alterations in hepatic metabolism of adult male rats following exposure to hydroxyprogesterone during embryonic development. Asian J Androl. 2006;8(4):463-7.

20. FDA. http://www.fda.gov/NewsEvents/Newsroom/PressAnnouncements/ ucm 149575.htm 